\title{
Initial introduction of robot-assisted, minimally invasive esophagectomy using the microanatomy-based concept in the upper mediastinum
}

\author{
Yasuhiro Shirakawa ${ }^{1,2}$ (D) Kazuhiro Noma ${ }^{1} \cdot$ Tomoyoshi Kunitomo $^{1} \cdot$ Masashi Hashimoto ${ }^{1}$. Naoaki Maeda ${ }^{1}$ \\ Shunsuke Tanabe ${ }^{1} \cdot$ Kazufumi Sakurama $^{1} \cdot$ Toshiyoshi Fujiwara $^{1}$
}

Received: 8 July 2020 / Accepted: 4 November 2020 / Published online: 10 November 2020

(c) Springer Science+Business Media, LLC, part of Springer Nature 2020

\begin{abstract}
Background We have recently standardized upper mediastinal lymph node dissection (UMLND) using a microanatomy-based concept in thoracoscopic esophagectomy in the prone position (TEPP), and introduced robot-assisted minimally invasive esophagectomy (RAMIE) using the same concept as in TEPP while aiming at solo surgery. The purpose of this study was to investigate the outcomes of RAMIE using the microanatomy-based concept in the initial introduction phase.

Methods We have performed more than 500 TEPP procedures as minimally invasive esophagectomy (MIE). After performing about 400 cases of MIE, we established a microanatomy-based standardization of UMLND. In October 2018, we introduced RAMIE, and have performed 75 procedures in 20 months. Two groups were analyzed: a group after microanatomy-based standardization in TEPP (100 cases after completing 400 cases of TEPP) and a RAMIE group ( 75 cases). Finally, 51 paired cases were matched using a propensity score. Furthermore, the change in postoperative short-term outcome for RAMIE in the initial introduction phase was analyzed.

Results Although there were no significant differences between the two groups in the number of upper mediastinal lymph nodes dissected, there was a significant decrease $(P=0.036)$ in intraoperative blood loss volume with RAMIE, representing a definite benefit for patients. The thoracoscopic operative time for RAMIE decreased by almost $100 \mathrm{~min}$ following less than 50 cases of experience, reaching the same level as that for recent TEPP, but with only one-tenth the operator experience. There were no significant differences in the total postoperative morbidity rate including the recurrent laryngeal nerve palsy rate. Conclusion RAMIE has been introduced safely and smoothly using the microanatomy-based concept established in TEPP.
\end{abstract}

Keywords Esophageal cancer $\cdot$ Microanatomy $\cdot$ Robot-assisted minimally invasive esophagectomy $\cdot$ Thoracoscopic esophagectomy $\cdot$ Upper mediastinal lymph node dissection

Esophageal cancer is the sixth leading cause of death from malignancy and is common in Asian countries including Japan, with squamous cell carcinoma seen in the majority of these countries. Esophageal cancer tends to metastasize easily and to have low 5-year survival rates (ranging from 15-25\%) [1]. Radical esophagectomy with extended

Yasuhiro Shirakawa

yasuwr@md.okayama-u.ac.jp

1 Department of Gastroenterological Surgery, Okayama University Graduate School of Medicine, Dentistry and Pharmaceutical Sciences, Okayama, Japan

2 Department of Surgery, Hiroshima City Hiroshima Citizens Hospital, 7-33 Motomachi, Naka-ku, Hiroshima 730-8518, Japan lymphadenectomy following neoadjuvant treatment is the primary curative treatment [2-5], but it is one of the most invasive surgeries [6-8]. In this surgery, upper mediastinal lymph node dissection (UMLD) is not only the most important process for improving the postoperative prognosis, but also the most difficult, even in the traditional open approach. In the past decades, several minimally invasive esophagectomy (MIE) procedures have been introduced in order to reduce surgical invasion. Thoracoscopic esophagectomy was introduced in the early 1990s [9]. Currently, thoracoscopic esophagectomy in the prone position (TEPP) has become the most common procedure in MIE because of the advantage of a good surgical field easily provided by gravity, an artificial pneumothorax, and good surgeon ergonomics. Of course, UMLND is the most important process even in 
TEPP. However, it is considered that UMLND in TEPP is more difficult than in conventional open esophagectomy or thoracoscopic esophagectomy in the lateral decubitus position (TELP). This is true especially for the left side, because a tangential surgical procedure while getting over the trachea is needed. To solve that difficulty, not only there is surgical field exposure by an assistant helpful, but there have been reports of technical innovations to effectively dissect the lymphatic chain around the recurrent laryngeal nerve [10-12]. Furthermore, some microanatomy-based concepts for UMLND using thoracoscopic magnified views have also been reported [13-16]. Recently, we established a microanatomy-based standardization of UMLND using thoracoscopic high-definition (HD) views with a focus on the meso-esophagus and reported its usefulness [17].

Robot-assisted minimally invasive esophagectomy (RAMIE) using the da Vinci system (Intuitive Surgical Inc., Sunnyvale, CA, USA) was introduced in the early 2000s. Although RAMIE has been considered to be an alternative surgical option for MIE, it is based on the TEPP technique. There have been several reports on its advantages in esophagectomy and reconstruction such as by Mckeown and Ivor Lewis due to its $10 \times$ magnified three-dimensional (3D) stereoscopic vision system, wrist-like joint system, and shake reduction system [18-21]. However, reports of its usefulness in UMLND have been rare, and the first worldwide, prospective, randomized, controlled study has just started [22]. The progress in the development of the robot system has also been remarkable. In the current da Vinci Xi system, there is no need to redock the system, and four flexible arms are available. We started RAMIE using the da Vinci Xi system in October 2018 and have performed 75 procedures in 20 months. Since the early phase of introduction, we have applied a microanatomy-based concept for the upper mediastinum devised in TEPP to RAMIE. Furthermore, we have attempted to perform a solo-surgery procedure making full use of the four robotic arms in the da Vinci Xi system.

The primary objective of this retrospective study was to investigate the intraoperative and postoperative short-term outcomes of the initial RAMIE procedures, aiming for solo surgery while using the same microanatomy-based concept as TEPP with a special focus on UMLND.

\section{Materials and methods}

\section{Patients}

TEPP was performed for 500 cases ( 430 males, 70 females) of esophageal cancer at Okayama University Hospital from June 2011 to April 2019, excluding cases of RAMIE. After performing about 350 procedures by April 2017, we attempted to standardize UMLND based on microanatomy using a magnified view. We established this standardized procedure after performing about 400 procedures by November 2017. In October 2018, RAMIE was introduced, and 75 procedures were performed by June 2020. In this study, two groups were analyzed: a group after microanatomy-based standardization in TEPP (100 cases after completing 400 TEPP procedures) and a RAMIE group (75 cases). After cases with tumor invading surrounding organs (T4), cases with omission of UMLND, and cases after thoracotomy were excluded, 51 paired cases were eventually matched using propensity score matching (Fig. 1). In this study, the clinicopathological factors, intraoperative and postoperative factors, and treatment outcomes for these two groups were examined.

This study was approved by the Ethics Committee of Okayama University Hospital (2001-2016).

\section{Operative procedure}

In this study, all surgeries were performed by two endoscopic surgical skill qualification system-qualified console surgeons in the same one team. The operative procedures of TEPP were performed as described in our previous reports [17, 23]. In RAMIE, the da Vinci Xi system has been used since the first case. The patient was immobilized in a prone position after endotracheal intubation using a single-lumen endotracheal tube and a bronchial blocker, as in TEPP. The ports for the 1st, 2nd, 3rd, and 4th arms of the da Vinci $\mathrm{Xi}$ system were placed at the 9th intercostal space (ICS) at the inferior scapular line, the 7th ICS at the posterior axillary line, the 5th ICS at the posterior axillary line, and the 3rd ICS at the mid-axillary line, respectively. Furthermore, the port for an assistant was placed at the 6th ICS, slightly anterior to the mid-axillary line. A thoracoscope with a 30-degree angle was mainly used in RAMIE, and an 8 to $10 \mathrm{mmHg}$ artificial pneumothorax using carbon dioxide $\left(\mathrm{CO}_{2}\right)$ was induced without one-lung ventilation. The primary devices for the operator in RAMIE were Maryland forceps and sharp scissors (Pott's scissors), and the tissues were coagulated and cut using the bipolar cutting method using the Maryland forceps [24].

\section{Microanatomy-based procedure in UMLD in RAMIE aiming for solo surgery}

The microanatomy-based concept devised in TEPP was applied to RAMIE, and a solo-surgery procedure in UMLND was established making full use of the four robotic arms of the da Vinci Xi system. In the RAMIE procedure, the mesoesophagus and visceral sheath are important. The mesoesophagus contains the lymph nodes around the recurrent laryngeal nerve, and the visceral sheath wraps the esophagus, trachea, and bilateral meso-esophagus [17]. First, the 


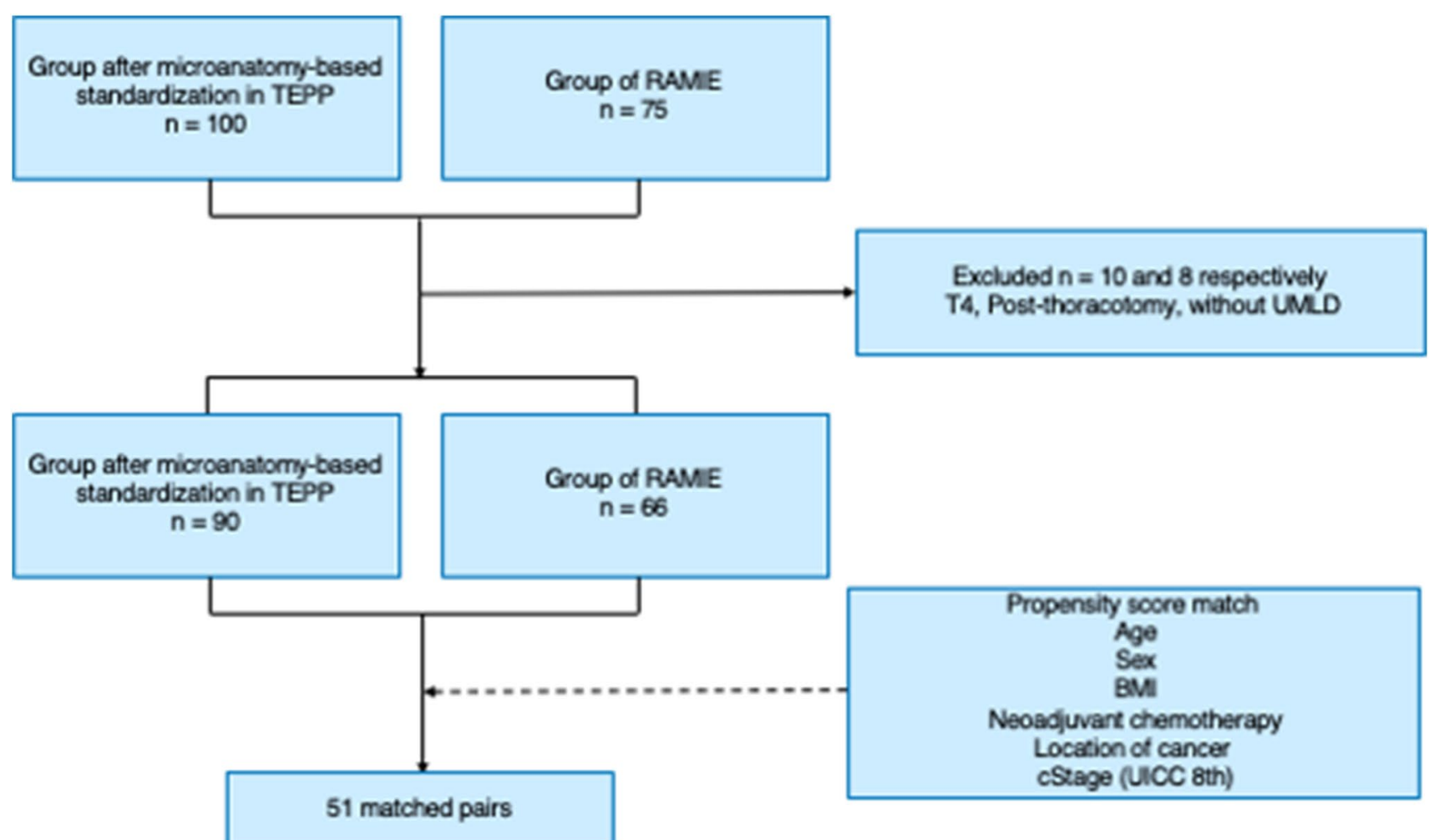

Fig. 1 Flow chart for patient and control group selection. TEPP, Thoracoscopic esophagectomy in the prone position; RAMIE, Robot-assisted minimally invasive esophagectomy; T4, Invasion of adjacent organs; $U M L D$, Upper mediastinal lymph node dissection; $B M I$, Body mass index

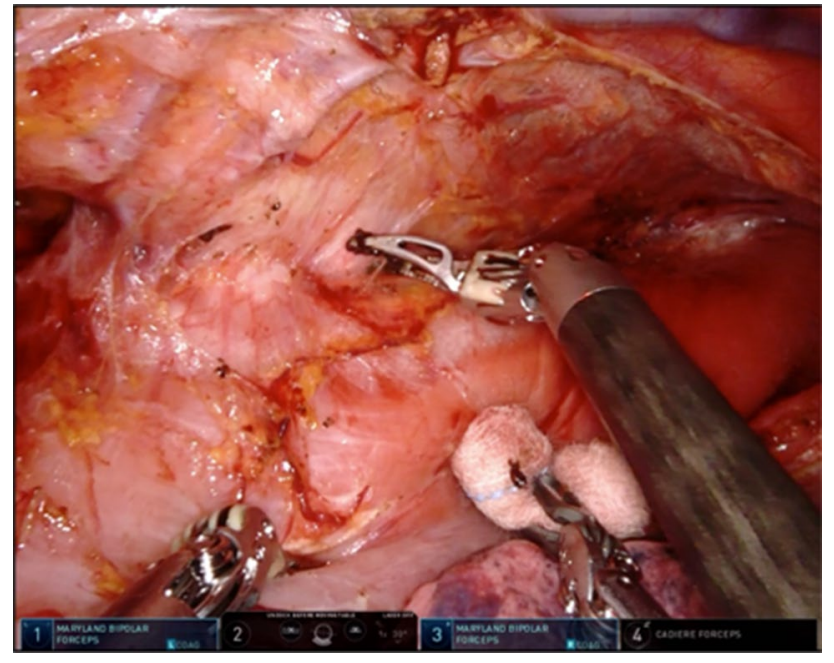

Fig. 2 Detachment of the visceral sheath on the left side while preserving the visceral sheath and rotating the esophagus and trachea using the 4th arm holding a bale-shaped gauze

dorsal and lateral sides of the visceral sheath are peeled off while rotating the esophagus and trachea using the 4th arm holding a bale-shaped gauze (Fig. 2). During this series of UMLND procedures, especially on the left side, an organ retractor (B. Braun, Tokyo, Japan) is used with a thread to pull up the esophagus to the dorsal side (Fig. 3). We consider that the organ retractor acts as a 5th arm in RAMIE and pulls out the thread through the chest wall to maintain moderate tension. Next, the esophagus and meso-esophagus are detached from the trachea, and the lymphatic chain is aggregated to the esophageal side, while the 4th arm presses and fixes the trachea. Eventually, lymph node dissection along the recurrent laryngeal nerve is performed from the central part to the peripheral part. The lymphatic chain is flipped up on the inner surface of the visceral sheath and slid down the nerve to its natural position while the surgical field is kept stable by these procedures (Fig. 4).

\section{Description and statistical analysis}

Clinicopathological factors were noted with reference to the Japanese Classification of Esophageal Cancer [25, 26] and the Union for International Cancer Control (UICC) Tumor Node Metastasis (TNM) Classification of Malignant Tumors, 8th edition [27]. Postoperative complications were categorized using the Clavien-Dindo classification [28]. A propensity score analysis was performed to compensate for differences in baseline characteristics between the pre-standardization group and the post-standardization group. Using a 

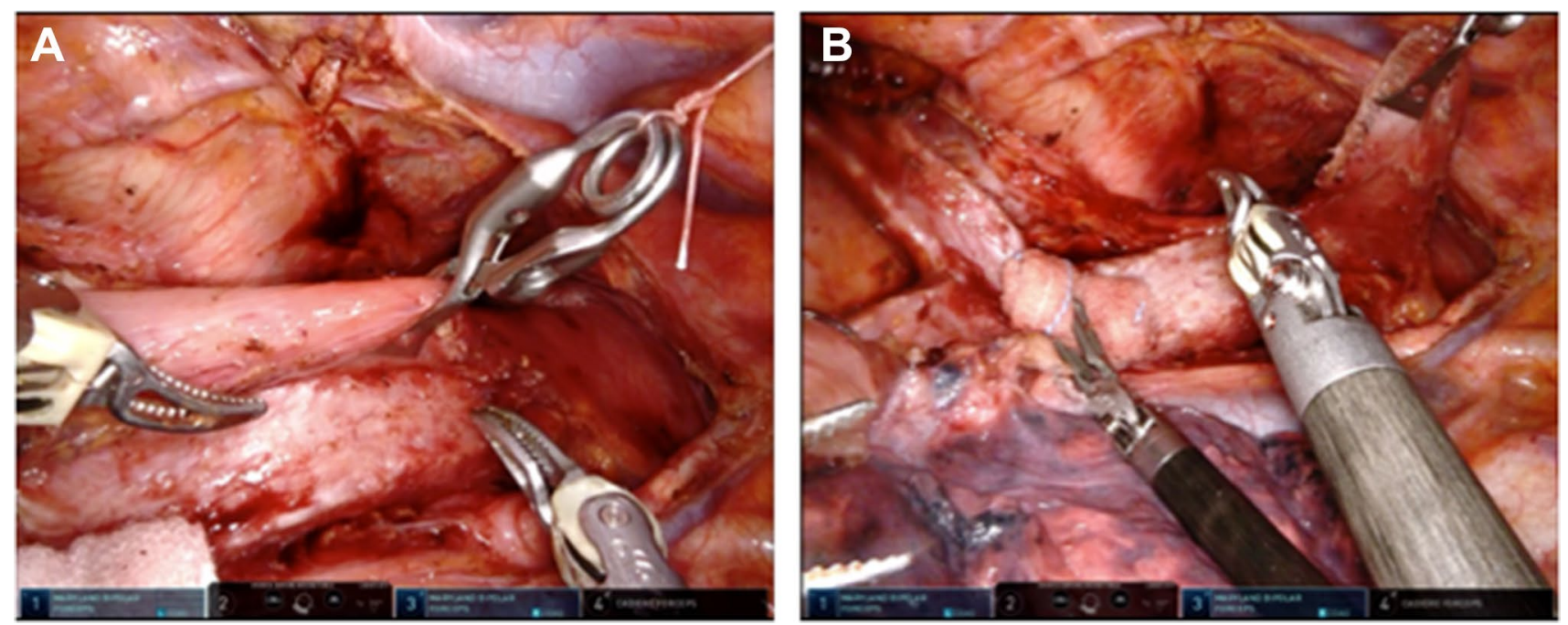

Fig. 3 Pulling up the esophagus using an organ retractor both before and after the transection of the esophagus, which acts like a 5th arm of RAMIE. RAMIE, Robot-assisted minimally invasive esophagectomy

logistic regression model that included age, sex, body mass index (BMI), neoadjuvant chemotherapy, location of cancer, and clinical (c) TNM, propensity scores were calculated as the conditional probability of receiving cases from either group. Eventually, a matched cohort of 51 pairs of cases was created from the two groups (Table 1). To evaluate the differences between the two groups, continuous variables were assessed using the Mann-Whitney test, and categorical variables were assessed using the $\chi^{2}$ test or Fisher's exact test. Differences were considered significant when the $P$ value was $<0.05$. Kaplan-Meier analyses were also used to estimate the cumulative survival of patients. All analyses were performed using JMP version 14 statistical analysis software (SAS Institute, Cary, NC, USA). The thoracoscopic operative time learning curve was analyzed using the moving average method [29, 30]. With the moving average method using the mean thoracoscopic operative time, the trends were clarified, and the changes were smoothed. A 5-case moving average was used in this study, and the exclusion criteria for the cases were the same as above.

\section{Results}

\section{Intraoperative findings}

Before propensity score matching, there was a significant difference in tumor location between the groups after microanatomy-based standardization in TEPP and the RAMIE group $(P=0.016)$. However, there were no significant differences in patient background characteristics between the two groups after propensity score matching (Table 1). No significant differences were found between the two groups with regard to the number of dissected No. 106 lymph nodes. Although no transfusion was performed in either group, the amount of intraoperative blood loss was significantly lower in the RAMIE group $(P=0.036)$. The thoracoscopic operative time for RAMIE decreased by almost $100 \mathrm{~min}$ following less than 50 cases of experience, reaching the same level as that for recent TEPP, but with only one-tenth the operator experience (Fig. 5). None of the patients in either group required conversion to thoracotomy (Table 2). We calculated the financial data regarding the surgical instruments in the first 5 cases of RAMIE. It showed that the cost of surgical instruments was $37.6 \%$ higher than conventional TEPP. However, also the increased cost was able to be covered by the national health insurance.

\section{Postoperative findings}

The weaning from the ventilator was completed within postoperative day one in all cases of both groups. There were no significant differences in the total morbidity rate or the incidence of respiratory complications or anastomotic leakage ( $\geq$ Grade 2 ). Regarding recurrent laryngeal nerve palsy, laryngoscopic assessment was performed on postoperative day (POD) 1, and palsy was defined as any dysmotility in the vocal cords, and no significant differences were found between the two groups with regard to the incidence of recurrent laryngeal nerve palsy ( $\geq$ Grade 1 ) (Table 2 ). There was no mortality in either group. Regarding the postoperative course, there were no significant differences in ICU stay or postoperative hospital stay. Furthermore, there was no postoperative mortality in either group. 


\section{Right side}

A

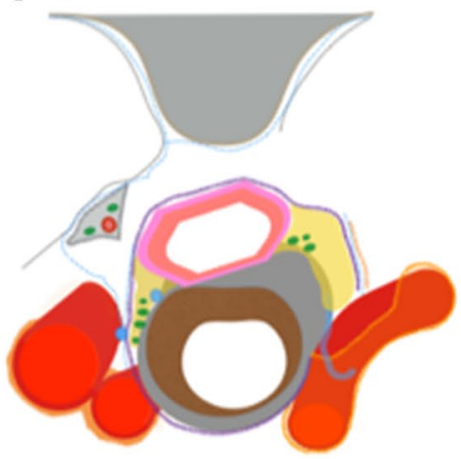

D

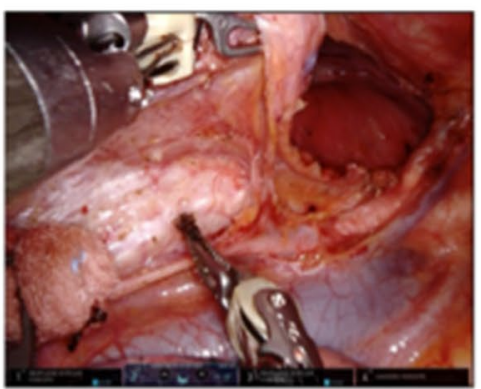

B

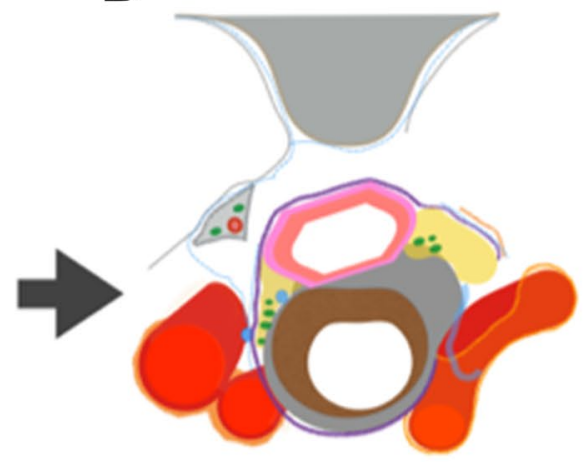

E

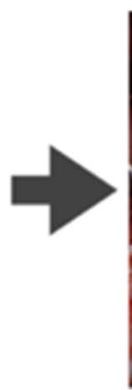

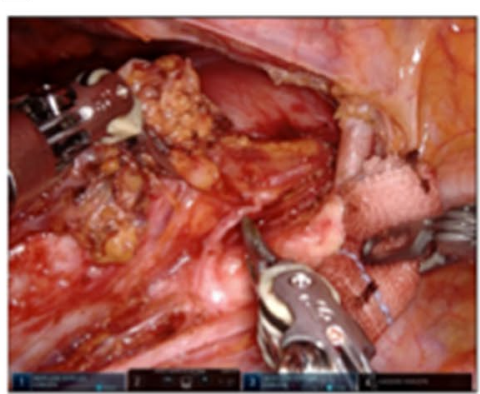

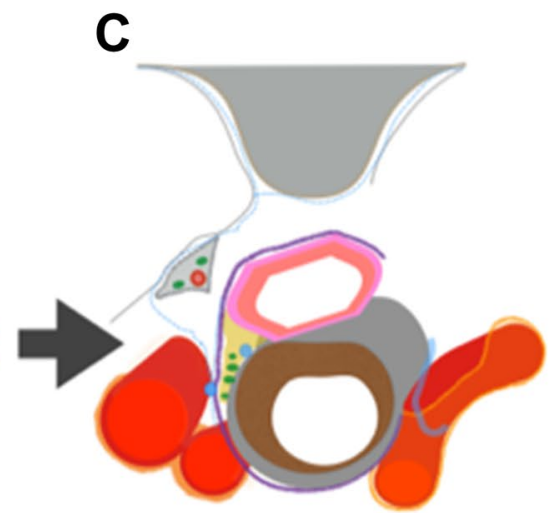

$\mathbf{F}$

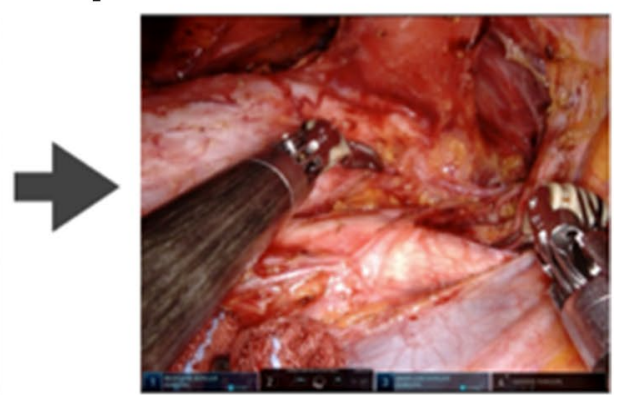

\section{Left side}
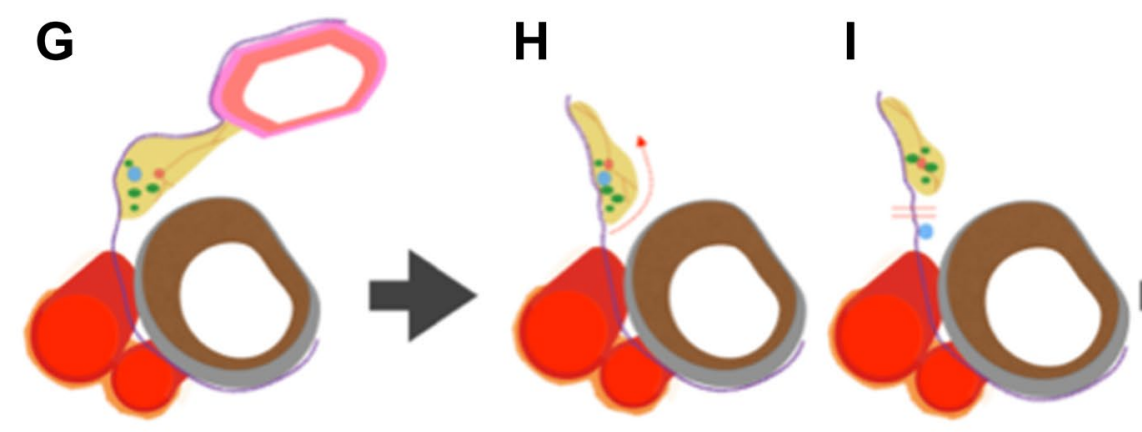

$\mathbf{J}$

K
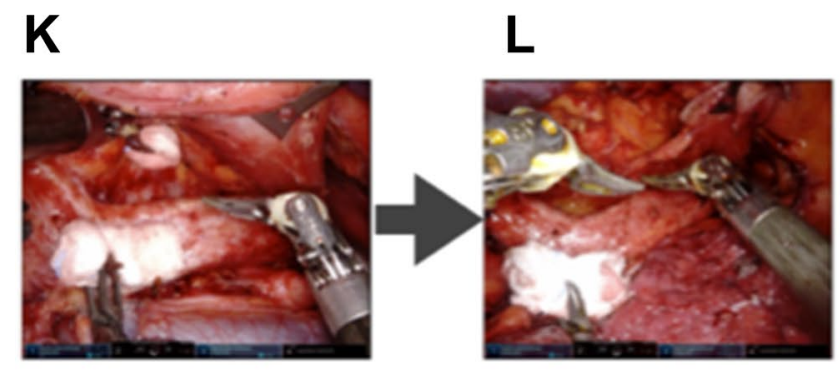

Fig. 4 A, D, G, K Detachment of the esophagus together with the lymphatic chain from the trachea and aggregation of the lymphatic chain to the esophagus side. B, E, H, I, L, M Identification of the left recurrent laryngeal nerve and lymph node dissection around it using
M

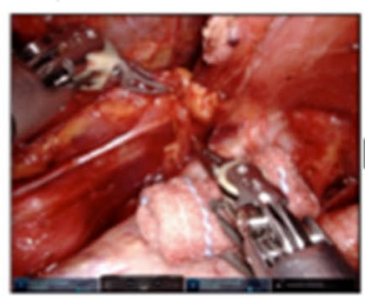

$\mathbf{N}$

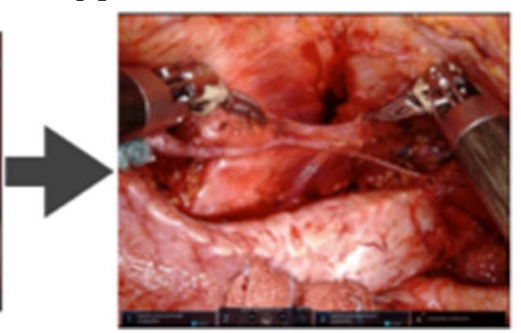

Maryland forceps or Pott's scissors. C, F, J, N The final findings after completing the upper mediastinal lymph node dissection after the microanatomy-based standardization in RAMIE. RAMIE Robotassisted minimally invasive esophagectomy 
Table 1 Patient characteristics before and after propensity matching

\begin{tabular}{|c|c|c|c|c|c|c|}
\hline \multirow[t]{2}{*}{ Characteristics } & \multicolumn{2}{|l|}{ Total cohort } & \multirow[t]{2}{*}{$P$ value } & \multicolumn{2}{|c|}{ Propensity score match cohort } & \multirow[t]{2}{*}{$P$ value } \\
\hline & $\begin{array}{l}\text { Group after microanat- } \\
\text { omy-based standardiza- } \\
\text { tion in TEPP }(n=90)\end{array}$ & $\begin{array}{l}\text { Group of RAMIE ( } 66 \\
\text { cases) }\end{array}$ & & $\begin{array}{l}\text { Group after microanat- } \\
\text { omy-based standardiza- } \\
\text { tion in TEPP }(n=51)\end{array}$ & $\begin{array}{l}\text { Group of RAMIE ( } 51 \\
\text { cases) }\end{array}$ & \\
\hline $\begin{array}{l}\text { Age, median [year } \\
\text { (IQR)] }\end{array}$ & $67(61-73)$ & $68(59-73)$ & $0.600^{\mathrm{a}}$ & $67(62-73)$ & $69(60-74)$ & $0.728^{\mathrm{a}}$ \\
\hline \multicolumn{7}{|l|}{ Gender } \\
\hline Male $(\%)$ & $74(82.2)$ & $56(84.8)$ & \multirow[t]{2}{*}{$0.664^{\mathrm{b}}$} & $44(86.3)$ & $43(84.3)$ & \multirow[t]{2}{*}{$0.780^{\mathrm{b}}$} \\
\hline Female $(\%)$ & $16(17.8)$ & $10(15.2)$ & & $7(13.7)$ & $8(15.7)$ & \\
\hline $\begin{array}{l}\text { BMI, median }[\mathrm{kg} / \mathrm{m} 2 \\
\text { (IQR)] }\end{array}$ & $21.9(20.2-23.4)$ & $21.8(19.6-24.0)$ & $0.577^{\mathrm{a}}$ & $22.5(19.9-23.3)$ & $21.4(19.5-23.5)$ & $0.301^{\mathrm{a}}$ \\
\hline $\begin{array}{l}\text { Neoadjuvant chemo- } \\
\text { therapy }(\%)\end{array}$ & $51(56.7)$ & $30(45.4)$ & $0.166^{\mathrm{b}}$ & $19(37.3)$ & $26(51.0)$ & $0.163^{\mathrm{b}}$ \\
\hline \multicolumn{7}{|l|}{ Tumor location } \\
\hline $\mathrm{Ce}(\%)$ & $6(6.7)$ & $3(4.6)$ & \multirow[t]{5}{*}{$0.016^{\mathrm{b}}$} & $3(5.9)$ & $3(5.9)$ & \multirow[t]{5}{*}{$0.764^{\mathrm{b}}$} \\
\hline Ut (\%) & $20(22.2)$ & $13(19.7)$ & & $7(13.7)$ & $12(23.5)$ & \\
\hline Mt (\%) & $40(44.4)$ & $22(33.3)$ & & $26(51.0)$ & $21(41.2)$ & \\
\hline $\operatorname{Lt}(\%)$ & $20(22.2)$ & $13(19.7)$ & & $13(25.5)$ & $13(25.5)$ & \\
\hline $\mathrm{Ae}(\%)$ & $4(4.4)$ & $15(22.7)$ & & $2(3.9)$ & $2(3.9)$ & \\
\hline \multicolumn{7}{|l|}{ Clinical stage (UICC 8th) } \\
\hline $0, \mathrm{I}, \mathrm{II}(\%)$ & $52(57.8)$ & $41(62.1)$ & \multirow[t]{2}{*}{$0.585^{\mathrm{b}}$} & $36(70.6)$ & $32(62.8)$ & \multirow[t]{2}{*}{$0.401^{\mathrm{b}}$} \\
\hline III, IV (\%) & $38(42.2)$ & $25(37.9)$ & & $15(29.4)$ & $19(37.2)$ & \\
\hline \multicolumn{7}{|l|}{ ASA-PS } \\
\hline $1(\%)$ & & & & $10(19.6)$ & $12(23.6)$ & \multirow[t]{3}{*}{$0.341^{\mathrm{b}}$} \\
\hline $2(\%)$ & & & & $31(60.8)$ & $35(68.6)$ & \\
\hline $3 \leqq(\%)$ & & & & $10(19.6)$ & $4(7.8)$ & \\
\hline \multicolumn{7}{|l|}{ Histological diagnosis } \\
\hline $\operatorname{SCC}(\%)$ & & & & $47(91.2)$ & $45(88.2)$ & \multirow[t]{3}{*}{$0.701^{\mathrm{b}}$} \\
\hline $\operatorname{ADC}(\%)$ & & & & $2(3.9)$ & $2(3.9)$ & \\
\hline Others $(\%)$ & & & & $2(3.9)$ & $4(7.9)$ & \\
\hline \multicolumn{7}{|l|}{ Lymph node dissection } \\
\hline $\begin{array}{l}\text { Two-field dissection } \\
\text { (\%) }\end{array}$ & & & & $19(37.2)$ & $24(47.1)$ & \multirow[t]{2}{*}{$0.316^{\mathrm{b}}$} \\
\hline $\begin{array}{l}\text { Three-field dissection } \\
(\%)\end{array}$ & & & & $32(62.8)$ & $27(52.9)$ & \\
\hline
\end{tabular}

$I Q R$ inter quartile rate, $B M I$ body mass index, $C e$ cerbical esophagus, $U t$ upper thoracic esophagus, $M t$ middle thoracic esophagus, $L t$ lower thoracic esophagus, $A e$ abdominal esophagus, $S C C$ squamous cell carcinoma, $A D C$ adenocarcinoma

${ }^{a}$ Mann-Whitney test

${ }^{\mathrm{b}} \chi^{2}$ test

\section{Discussion}

Almost 20 years have passed since RAMIE was introduced in clinical practice, and much progress has been made with the introduction of new technologies: a $10 \times$ magnified $3 \mathrm{D}$ view, a shake reduction system, and a wrist-like joint system. There have been reports of the benefits of Westernstyle esophageal cancer surgeries, such as by Mckeown and Ivor Lewis [18-21]. Since the introduction of the new da Vinci Xi system, in which four flexible arms are available and redocking is not needed during surgery, the number of
RAMIE cases and the interest in this procedure have been increasing more and more. Especially in Japan, the tendency has been more marked after 2018 when the national health insurance began to cover this surgery.

On the other hand, MIE has already had an almost 30-year history. Today, thoracoscopic esophagectomy has become the most common MIE procedure worldwide. Several standardizations of thoracoscopic esophagectomy accompanied by lymph node dissection have been reported [10-12]. Recently, we also published microanatomy-based standardization focusing on UMLND [17]. We thought that 


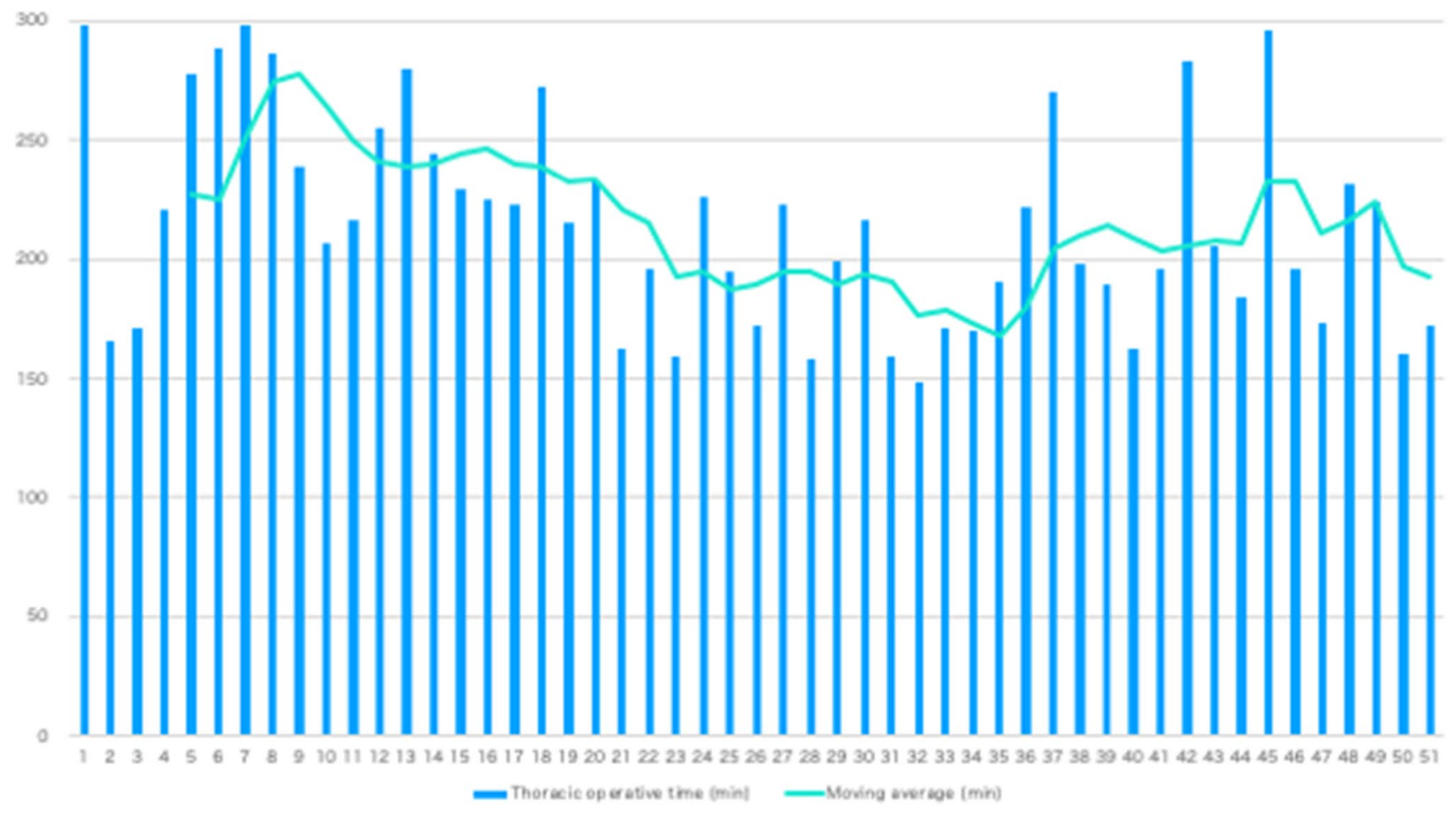

Fig. 5 Five-case moving average of thoracoscopic operative time

Table 2 Surgical findings

\begin{tabular}{|c|c|c|c|}
\hline Variables & $\begin{array}{l}\text { Group after microanatomy-based standardi- } \\
\text { zation in TEPP }(n=51)\end{array}$ & $\begin{array}{l}\text { Group of RAMIE ( } 51 \\
\text { cases) }\end{array}$ & $P$ value \\
\hline \multicolumn{4}{|l|}{ Intraoperative findings } \\
\hline Thoracoscopic operative time [min (IQR)] & $211(170-236)$ & $215(172-239)$ & $0.356^{\mathrm{a}}$ \\
\hline Blood loss [ml (IQR)] & $235(100-450)$ & $150(100-235)$ & $0.038^{\mathrm{a}}$ \\
\hline Number of dissected No. 106 lymph nodes (IQR) & $10(8-15)$ & $11(8-14)$ & $0.799^{\mathrm{a}}$ \\
\hline Conversion to thoracotomy (\%) & $0(0)$ & $0(0)$ & $1.000^{\mathrm{b}}$ \\
\hline \multicolumn{4}{|l|}{ Postoperative findings } \\
\hline Total morbidity [Grade II (\%)] & $25(49.0)$ & $23(45.1)$ & $0.692^{\mathrm{b}}$ \\
\hline Respiratory complication [Grade II $\sim(\%)]$ & $10(19.6)$ & $9(17.7)$ & $0.799^{\mathrm{b}}$ \\
\hline \multicolumn{4}{|l|}{ Recurrent laryngeal nerve palsy } \\
\hline$[$ Grade $\mathrm{I} \sim(\%)]$ & $8(15.7)$ & $9(17.7)$ & $0.791^{\mathrm{b}}$ \\
\hline Anastomotic leakage [Grade II $\sim(\%)]$ & $7(13.7)$ & $5(9.8)$ & $0.539^{\mathrm{b}}$ \\
\hline ICU stay [day (IQR)] & $6(5-6)$ & $5(5-6)$ & $0.502^{\mathrm{a}}$ \\
\hline Postoperative hospital stay [day (IQR)] & $23(18-33)$ & $25(21-36)$ & $0.097^{\mathrm{a}}$ \\
\hline In- hospital mortality (\%) & $0(0)$ & $0(0)$ & $1.000^{\mathrm{b}}$ \\
\hline
\end{tabular}

Complications are described on the Clavien-Dindo classification [29]

$I Q R$ inter quartile rate, $I C U$ intensive care unit

${ }^{\mathrm{a}}$ Mann-Whitney test

${ }^{\mathrm{b}} \chi^{2}$ test 
the standardized technique in thoracoscopic surgery could be applied to RAMIE. Indeed, in our initial introduction of RAMIE, the microanatomy-based procedures established in TEPP were successfully applied, given the intraoperative and postoperative short-term outcomes.

In RAMIE, the selection of instruments is vital because energy devices such as an ultrasonically vibrating scalpel or a vessel sealing system used in general thoracoscopic surgery could not be applied, and it took a slightly longer time to change devices than in usual thoracoscopic surgery. The primary energy devices used in RAMIE were Maryland forceps in both hands, and the tissues were coagulated and cut by the bipolar cutting method using Maryland forceps without changing instruments. The bipolar cutting method is advantageous because of the precise dissection by sharp forceps and the lower thermal damage to surrounding tissues [24]. This method also contributed to decreasing the use of hemostatic clips as much as possible. In addition, the fourth arm of the da Vinci Xi system is useful for creating an excellent surgical field, like an experienced assistant. It enabled us to proceed with solo surgery in almost all procedures.

We believe that these advantages of the da Vinci Xi system make it suitable for the complicated and troublesome UMLND in TEPP, and RAMIE should contribute to making it more precise, more accessible, and safer. The wrist-like joints and shake reduction system under a 3D magnified view are very useful, especially for lymph node dissection along the left recurrent laryngeal nerve that requires entering above the trachea. Using those advantages, the robotic arms could be used more intuitively than the instruments in the conventional TEPP and could overcome the lack of optic and tactile feedback. Although we are doing RAMIE applying TEPP's concept and technology, it feels more like the sensation of open surgery than the sensation of endoscopic surgery. In other words, we feel like doing surgery as if putting both small hands into the chest cavity or becoming a dwarf. We think that is the true advantage of the robotic platform. RAMIE has also reduced the stress felt by surgeons. The 4th arm of the da Vinci Xi system and the organ retractor also enabled us to perform solo surgery in that procedure. The 4th arm could rotate the trachea easily and safely and maintain the correct surgical field. The organ retractor connected by silk thread could grasp the esophagus gently and pull it up with adequate tension, and it could be removed and reattached anytime, like a 5 th arm.

Owing to those robotic technologies, we have standardized UMLND in RAMIE for solo surgery after gaining experience with just a small number of cases, and the thoracoscopic operative time for RAMIE decreased by almost 100 min. Furthermore, most postoperative short-term outcomes have reached nearly the same level as those achieved with recent TEPP procedures, but with only about onetenth the operator experience. The significant decrease in intraoperative blood loss volume is also a definite benefit of RAMIE. We had thought RAMIE would reduce the rate of recurrent nerve palsy rate. In results, there was no significant reduction of it compared with conventional TEPP. Although the robotic arms were very useful to keep the stable-wide surgical field and to perform the sharp lymph node dissection along the nerve, there might be a possibility that the nerve was stretched a little excessively resulted in the recurrent laryngeal nerve palsy. On the other hand, we think the da Vinci Xi system is also much useful to teach the techniques thanks to using the writable monitor or the double console system.

There were several limitations to this study. This was a retrospective, small cohort study conducted at a single center with analysis only of cases performed by senior surgeons who began RAMIE as endoscopic surgical skill qualification system-qualified surgeons. Thus, the benefits of RAMIE, especially the reduction of operative time, might be simply owing to the skills and experience gained with TEPP. To verify whether RAMIE is actually useful, the short and longterm outcomes for younger surgeons beginning TEPP and RAMIE should be analyzed in a prospective, randomized study, if possible. The recognition of new microanatomy brought by these technologies will contribute to the further improvement of endoscopic surgery. Thus, they will also need to be evaluated for their usefulness.

In conclusion, the microanatomy-based concept in the upper mediastinum established in TEPP was useful for the safe introduction of RAMIE and more enhanced in RAMIE while enabling us to perform solo surgery.

\section{Compliance with ethical standards}

Disclosures Drs. Yasuhiro Shirakawa, Kazuhiro Noma, Tomoyoshi Kunitomo, Masashi Hashimoto, Naoaki Maeda, Shunsuke Tanabe, Kazufumi Sakurama, and Toshiyoshi Fujiwara have no conflicts of interest or financial ties to disclose.

Ethical approval All procedures followed were in accordance with the Helsinki Declaration of 1964 and its later versions. This study was approved by the Ethics Committee of Okayama University Hospital (2001-2016).

\section{References}

1. Parkin DM, Bray F, Ferlay J, Pisani P (2005) Global cancer statistics, 2002. CA Cancer J Clin 55:74-108

2. Akiyama H, Tsurumaru M, Kawamura T, Onu Y (1981) Principles of surgical treatment for carcinoma of the esophagus analysis of lymph node involvement. Ann Surg 194:438-445

3. Akiyama H, Tsurumaru M, Udagawa H, Kajiyama Y (1994) Radical lymph node dissection for cancer of the thoracic esophagus. Ann Surg 220:364-372 
4. Fujita H, Sueyoshi S, Tanaka T, Fujii T, Toh U, Mine T, Sasahara H, Sudo T, Matono S, Yamana H, Shirouzu K (2003) Optimal lymphadenectomy for squamous cell carcinoma in the thoracic esophagus: comparing the short- and long-term outcome among the four types of lymphadenectomy. World J Surg 27:571-579

5. Igaki H, Tachimori Y, Kato H (2004) Improved survival for patients with upper and/or middle mediastinal lymph node metastasis of squamous cell carcinoma of the lower thoracic esophagus treated with 3-field dissection. Ann Surg 239:483-490

6. Atkins BZ, Shah AS, Hutcheson KA, Mangum JH, Pappas TN, Harpole DH Jr, D'Amico TA (2004) Reducing hospital morbidity and mortality following esophagectomy. Ann Thorac Surg 78:1170-1176

7. Dimick JB, Pronovost PJ, Cowan JA, Lipsett PA (2003) Complications and costs after high-risk surgery: where should we focus quality improvement initiatives? J Am Coll Surg 196:671-678

8. Watson A (1994) Operable esophageal cancer: current results from the West. World J Surg 18:361-366

9. Cuschieri A (1994) Thoracoscopic subtotal oesophagectomy. Endosc Surg Allied Technol 2:21-25

10. Noshiro H, Iwasaki H, Kobayashi K, Uchiyama A, Miyasaka Y, Masatsugu T, Koike K, Miyazaki K (2010) Lymphadenectomy along the left recurrent laryngeal nerve by a minimally invasive esophagectomy in the prone position for thoracic esophageal cancer. Surg Endosc 24:2965-2973

11. Oshikiri T, Yasuda T, Harada H, Goto H, Oyama M, Hasegawa H, Ohara T, Sendo H, Nakamura T, Fujino Y, Tominaga M, Kakeji Y (2015) A new method (the "Bascule method") for lymphadenectomy along the left recurrent laryngeal nerve during prone esophagectomy for esophageal cancer. Surg Endosc 29:2442-2450

12. Ozawa S, Ito E, Kazuno A, Chino O, Nakui M, Yamamoto S, Shimada H, Makuuchi H (2013) Thoracoscopic esophagectomy while in a prone position for esophageal cancer: a preceding anterior approach method. Surg Endosc 27:40-47

13. Akagawa S, Hosogi H, Yoshimura F, Kawada H, Kanaya S (2018) Mesenteric excision for esophageal cancer surgery: based on the concept of mesotracheoesophagus. Int Cancer Conf J 7:117-120

14. Fujiwara H, Kanamori J, Nakajima Y, Kawano T, Miura A, Fujita T, Akita K, Daiko H (2018) An anatomical hypothesis: a "concentric-structured model" for the theoretical understanding of the surgical anatomy in the upper mediastinum required for esophagectomy with radical mediastinal lymph node dissection. Dis Esophagus 32:doy119

15. Osugi H, Narumiya K, Kudou K (2017) Supracarinal dissection of the oesophagus and lymphadenectomy by MIE. J Thorac Dis 9:S741-S750

16. Tokairin Y, Nakajima Y, Kawada K, Hoshino A, Okada T, Ryotokuji T, Okuda M, Kume Y, Kawamura Y, Yamaguchi K, Nagai K, Akita K, Kinugasa Y (2018) Histological study of the thin membranous structure made of dense connective tissue around the esophagus in the upper mediastinum. Esophagus 15:272-280

17. Shirakawa Y, Noma K, Maeda N, Tanabe S, Sakurama K, Fujiwara T (2020) Microanatomy-based standardization of left upper mediastinal lymph node dissection in thoracoscopic esophagectomy in the prone position. Surg Endosc. https://doi.org/10.1007/ s00464-020-07407-9

18. Chen J, Liu Q, Zhang X, Yang H, Tan Z, Lin Y, Fu J (2019) Comparisons of short-term outcomes between robot-assisted and thoraco-laparoscopic esophagectomy with extended two-field lymph node dissection for resectable thoracic esophageal squamous cell carcinoma. J Thorac Dis 11:3874-3880

19. Na KJ, Park S, Park IK, Kim YT, Kang CH (2019) Outcomes after total robotic esophagectomy for esophageal cancer: a propensitymatched comparison with hybrid robotic esophagectomy. J Thorac Dis 11:5310-5320

20. Tagkalos E, Goense L, Hoppe-Lotichius M, Ruurda JP, Babic B, Hadzijusufovic E, Kneist W, van der Sluis PC, Lang H, van Hillegersberg R, Grimminger PP (2019) Robot-assisted minimally invasive esophagectomy (RAMIE) compared to conventional minimally invasive esophagectomy (MIE) for esophageal cancer: a propensity-matched analysis. Dis Esophagus. https://doi. org/10.1093/dote/doz060

21. Van Der Sluis PC, Tagkalos E, Hadzijusufovic E, Babic B, Uzun E, Van Hillegersberg R, Lang H, Grimminger PP (2020) Robotassisted minimally invasive esophagectomy with intrathoracic anastomosis (ivor lewis): promising results in 100 consecutive patients (the European experience). J Gastrointest Surg. https:// doi.org/10.1007/s11605-019-04510-8

22. Chao Y-K, Li Z-G, Wen Y-W, Kim D-J, Park S-Y, Chang Y-L, Van Der Sluis PC, Ruurda JP, Van Hillegersberg R (2019) Roboticassisted Esophagectomy vs Video-Assisted Thoracoscopic Esophagectomy (REVATE): study protocol for a randomized controlled trial. Trials. https://doi.org/10.1186/s13063-019-3441-1

23. Shirakawa Y, Noma K, Maeda N, Katsube R, Tanabe S, Ohara T, Sakurama K, Fujiwara T (2014) Assistant-based standardization of prone position thoracoscopic esophagectomy. Acta Med Okayama 68:111-117

24. Suda K, Nakauchi M, Inaba K, Ishida Y, Uyama I (2016) Robotic surgery for upper gastrointestinal cancer: current status and future perspectives. Dig Endosc 28:701-713

25. Japanese Classification of Esophageal Cancer (2017a) 11th Edition: part I. Esophagus 14:1-36J

26. Japanese Classification of Esophageal Cancer (2017b) 11th Edition: part II and III. Esophagus 14:37-65

27. Brierley JD, Gospodarowicz MK, Wittekind C (2017) TNM classification of malignant tumors (UICC international union against cancer), 8th edn. Wiley-Blackwell, Oxford

28. Dindo D, Demartines N, Clavien PA (2004) Classification of surgical complications: a new proposal with evaluation in a cohort of 6336 patients and results of a survey. Ann Surg 240:205-213

29. Kayano H, Okuda J, Tanaka K, Kondo K, Tanigawa N (2011) Evaluation of the learning curve in laparoscopic low anterior resection for rectal cancer. Surg Endosc 25:2972-2979

30. Yamamoto M, Okuda J, Tanaka K, Kondo K, Asai K, Kayano H, Masubuchi S, Uchiyama K (2013) Evaluating the learning curve associated with laparoscopic left hemicolectomy for colon cancer. Am Surg 79:366-371

Publisher's Note Springer Nature remains neutral with regard to jurisdictional claims in published maps and institutional affiliations. 